\title{
IMAGE FUSION WITH THE HERMITE TRANSFORM
}

\author{
Boris Escalante-Ramirez and Alejandra. López-Caloca \\ Center for Geography and Geomatics Research, "Ing. Jorge L. Tamayo", A.C. \\ Blvd. Cataratas No.3, Jardines del Pedregal, México, D.F., 01900 \\ National University of México, School of Engineering, Graduate Division \\ Apdo. Postal 70-256, México, D.F., 04510 \\ boris@servidor.unam.mx, alopez@centrogeo.org.mx
}

\begin{abstract}
The Hermite Transform is an image representation model that incorporates some important properties of visual perception such as the analysis through overlapping receptive fields and the Gaussian derivative model of early vision. It also allows the construction of pyramidal mutirresolution analysis-synthesis schemes. We show how the Hermite Transform can be used to build image fusion schemes that take advantage of the fact that Gaussian derivatives are good operators for the detection of relevant image patterns at different spatial scales. These patterns are later combined in the transform coefficient domain. Applications of this fusion algorithm are found in medical imagery and remote sensing, name.
\end{abstract}

\section{INTRODUCTION}

Image fusion has become a useful tool to enhance information provided by two or more sensors by combining the most relevant features of each image. A wide range of disciplines including remote sensing and medicine, have taken advantage of fusion techniques which in recent years have evolved from simple linear combinations to sophisticated methods based on principal components, color models and wavelets among others [6]. Information in the fused image must lead to improved accuracy (from redundant information) and improved capacity (from complementary information). Moreover, from a visual perception point of view, patterns included in the fused image must be perceptually relevant and must not include distracting artifacts. Our approach aims at analyzing images by means of an image representation model that allows us to identify perceptual relevant patterns to be included in the fusion process while discriminating spurious artifacts. Human viewers often assess the quality of the results of image processing. In such cases, errors are significant only if perceived. Therefore, with a deep understanding of the human visual system (HVS) their properties can be exploited to achieve resulting images that look better.

The Gabor model is one of the most widely used in HVS modeling at the visual cortex level [1], [7]. A filter design-based approach has been also of interest in modeling the processing by the visual cortex. The Cortex transform [8] is one of these types of image transform whose analysis functions were designed to approximate the Gabor shape with setable frequency and orientation bandwidths. An alternative model proposed by Young in 1987 is based on the Gaussian derivatives (GDs) [10]. Young showed that these functions model the measured receptive field data more accurately than the Gabor functions do [9]. Like the receptive fields, both Gabor functions and GD are spatially local and they consist of alternating excitatory and inhibitory regions in a decaying envelope. However, the GD analysis is found to be more efficient because it takes advantage of the fact that GDs comprise an orthogonal basis if they belong to the same point of analysis. In related work, the GDs have been interpreted as the product of Hermite polynomials and a Gaussian window [5], where windowed images are decomposed into a set of Hermite polynomials.

\section{HERMITE TRANSFORM}

This transform is a particular case of a family of transformations known as polynomial transforms [5] with a basic difference in the choice of the window function $w(x)$. For the case we are interested in, the window corresponds to a Gaussian function, defined in one dimension by

$$
w^{2}(x)=\frac{1}{\sigma \sqrt{\pi}} e^{-(x / \sigma)^{2}}
$$

with scale parameter $\sigma>0$. In the analysis stage, the input signal $f(x)$ is firstly localized by applying the window $w(x$ - $m T$ ) at positions $m T$, for $m$ integer and $T>0$. Then, the portion of the signal within the window, is expanded on 
the basis $\left\{\rho_{n}(x-m T) w(x-m T), n=0,1,2, \ldots, \infty ; m \in Z\right\}$, with

$$
\rho_{n}(x)=\frac{1}{\sqrt{2^{n} n !}} H_{n}(x / \sigma)
$$

for $n=0,1,2, \ldots, \infty$, where $H_{n}(x)$ denotes the Hermite polynomial of order $n$ [5]. The expansion is expressed under general conditions as

$$
w(x-m T)\left[f(x)-\sum_{n=0}^{\infty} f_{n}[m] \rho_{n}(x-m T)\right]=0
$$

where the Hermite coefficients $f_{n}[m]$, for $n=0,1,2, \ldots, \infty$ and $m \in Z$, are given by

$$
f_{n}[m]=\int_{-\infty}^{\infty} f(x) \rho_{n}(x-m T) w^{2}(x-m T) d x
$$

which is equivalent to sample the output of the convolution of the signal with the decimation filters $d_{n}(x)$ $=\rho_{n}(-x) w^{2}(-x)$, for $n=0,1,2, \ldots, \infty$, at points $m T, m \in Z$. The impulse response of these filters corresponds, except for a constant, to the Gaussian derivatives, since they can be written as

$$
d_{n}(x)=\frac{1}{\sqrt{2^{n} n !}} \frac{d^{n}}{d(x / \sigma)^{n}} w^{2}(x)
$$

Therefore, Hermite transform describes the input signal $f(x)$ in terms of its derivatives at a given scale for all position belonging to the sampling lattice $S=\{m T, m$ $\in Z, T>0\}$. While the signal $f(x)$ is synthesized from the coefficients $f_{n}[\mathrm{~m}]$ by integrating the local expansions of (1), provided that the weighting function

$$
W(x)=\sum_{m=-\infty}^{\infty} w^{2}(x-m T)
$$

is different from zero for all $x$, this is

$$
f(x)=\sum_{n=0}^{\infty} \sum_{m=-\infty}^{\infty} f_{n}[m] s_{n}(x-m T)
$$

with the interpolating filters $s_{n}(x)=\rho_{n}(x) w^{2}(x) / W(x)$, for $n$ $=0,1,2, \ldots, \infty$.

The expansion

$$
f_{w}(x-m T)=\sum_{n=0}^{\infty} f_{n}[m] d_{n}(m T-x)
$$

of the localized signal $f_{w}(x-m T)=f(x) w^{2}(x-m T)$, expresses the contribution of the coefficients $\{f\}_{m}=\left\{f_{n}[m], n=\right.$ $0,1,2, \ldots\}$ at position $m T$ to the over all synthesized signal. For a $D$-dimensional input signal we apply the same analysis and synthesis stages for each dimension, since a $D$-dimensional (isotropic) Gaussian window is defined by multiplying $D$ one-dimensional Gaussian windows with the same scale parameter $\sigma$.
In order to obtain a full understanding of the concept of scale, we look at an image as a representation of a physical scene. Such a representation is influenced by physical quantities that can be either measured or estimated. The influence of the viewing distance, for instance, can be interpreted as a scaling of the intensity distribution of our representation. This means that when moving away from the observed object we increase the scale of our representation and, therefore, some detail on the perceived image is lost, while getting closer reduces scale and then more detail can be perceived.

The viewing angle, as the relative position of the vision system, is another physical factor that influences the representation of visual patterns within the scene. Such viewing angle makes image structures to be perceived with certain orientation, which can only be seen by operators that are sensitive to orientation changes. From this, it is clear the need to insert an orientation parameter in the scale-space representation to obtain an orientation-scale-space representation.

\section{IMAGE FUSION}

\subsection{Directional analysis}

The proposed fusion algorithm works on a multiresolution fashion, which allows the detection and reconstruction of objects according to its scale space representation. Multiresolution is achieved through a pyramidal scheme by transforming the zero order coefficient of the previous layer. At each pyramidal level spurious information such as speckle is rejected while relevant information is kept. Considering that objects are composed of edges that can be detected at different spatial resolutions, we use the first order transform coefficients for this task. For the case of the Hermite Transform, these coefficients are obtained by convolving the image with a first derivative of a Gaussian along either one of the main orientations. This operator has long been recognized as a good edge detector. We improve the detection task by projecting the local two dimensional (2D) transform coefficients into one dimensional (1D) coefficients rotated to the direction of maximum energy. The k-order 1D polynomial coefficient oriented to direction $\theta$ is given by:

$$
K_{k, \theta}(p, q)=\sum_{n=0}^{k} \sum_{m=0}^{n} f_{m, n-m}[p, q] h_{k, \theta}(m, n-m)
$$

where $\theta$ is the orientation of the rotated 1D transform coefficient.

$h_{k, \theta}$ is an angle function that projects two dimensional coefficient $f_{m, n-m}$ into $\theta$ direction [3] [4].

In practice, we limit the number of considered orientations to eight, in agreement with the number degrees of freedom of the analysis function. Optimal $\theta$ 
orientation is estimated by maximizing the directional contrast $C_{\theta}$ defined by:

$$
C_{\theta}=\log \left[1+\sum_{k=1}^{N} K_{k, \theta}^{2}\right]
$$

For the reconstruction of patterns that are to be included in the fused image, only those that are oriented in the optimal orientation will be considered. In this way, artifacts and noise will be removed from edges and lines.

\subsection{Noise Removal}

In order to discriminate noise from relevant information, such as borders and lines, an energy mask containing image locations of these relevant patterns is built by thresholding the first-order transform coefficient energy $E_{l}$ :

$$
E_{1}=f_{0,1}^{2}+f_{1,0}^{2}
$$

where $f_{0,1}$ and $f_{1,0}$ are the first-order Hermite transform coefficients.

As stated before, the filters used to calculate these coefficients are good edge detectors. The threshold is set according to the desired percentage of noise reduction in the probability density function of the energy $E_{l}$. Assuming additive white noise with variance $\sigma_{l}^{2}$, the first order coefficient variance in homogeneous regions is given by:

$$
\sigma^{2}=\alpha \sigma_{l}^{2}
$$

where:

$$
\alpha=\left|R_{L}(x, y) * D_{10}(x, y) * D_{10}(-x,-y)\right|_{x=y=0}
$$

$R_{L}$ is the normalized autocorrelation function of the input noise, and $D_{1,0}$ is the filter used to calculate the first-order coefficients. Assuming Gaussian noise, the energy probability density function is exponential:

$$
P\left(E_{1}\right)=\frac{1}{2 \sigma^{2}} \exp \left(-\frac{E_{1}}{2 \sigma^{2}}\right)
$$

where: $\sigma^{2}$ is defined by (6)

Finally, the threshold is fixed:

$$
T=2 \ln \left(\frac{1}{P_{R}}\right) \sigma^{2}
$$

where $P_{R}$ is the probability (percentage) of noise left on the image and will be set by the user. For the case of multiplicative noise, such as the speckle of Synthetic Aperture Radar (SAR) images, an adaptive algorithm has been developed in order to set the threshold locally. A detailed description has been previously published [2].

\subsection{Coefficient fusion}

Directional analysis and noise removal is performed in each image to fuse. Thresholded energy masks are then analyzed using one of several standard criteria developed for transform-based fusion, such as maximum absolute value with or without constancy, etc. [6]. This procedure will indicate the locations where each coefficient set will contribute in the fused image. A single set of coefficients is obtained with this procedure. Inverse Hermite transform over these coefficients produces the fused image.

\section{RESULTS AND CONCLUSIONS}

Figure 1 shows the result of fusing a $30 \mathrm{~m}$ resolution band-1 LANDSAT 7 ETM+ image with a $8 m$ resolution RADARSAT image of Mexico City with the Hermite Transform method. Fusion with the wavelet transform is also showed in figure 1. Detailed inspection shows that the Hermite Transform provides a better image representation model for image fusion. Fused images show that the local directional analysis, and noise removal strategy help not only remove noise and artifacts, but also enhances the fused image. Since Hermite transform operators are based on Gaussian derivatives, its expansion coefficients are perceptually relevant and are very good suited to detect image patterns that are visually meaningful and that describe objects according to the Scale Space theory.

\section{ACKNOWLEDGEMENTS}

This work was supported by DGAPA-PAPIIT grant IN107101 and CONACyT grant 39636-F.

\section{REFERENCES}

[1] Bastiaans, M. "Gabor's signal expansion and degrees of freedom of a signal." Opt. Acta, vol. 29, pp 1223-1229, 1982.

[2] P. Camarillo-Sandoval, P.; Varela-López, A; and Escalante-Ramírez, B, "Adaptive Multiplicative-Noise Reduction in SAR Images with Polynomial Transforms", IGARSS'98, IEEE Geoscience and Remote Sensing Society, Proceedings, pp. 1171-1173, July 1998.

[3] Martens, J.-B. "Local orientation analysis in images by means of the Hermite transform" Image Processing, IEEE Transactions on Volume: 6, 8, pp. 1103 -1116, Aug. 1997.

[4] Martens, J.-B. "The Hermite transform-applications". IEEE Transactions on Acoustics, Speech and Signal Processing. Volume: 38, 9, pp. $1607-1618$, Sept. 1990.

[5] Martens, J.-B. "The Hermite transform-theory". IEEE Transactions on Acoustics, Speech and Signal Processing. Volume: 38, 9, pp. $1595-1606$, Sept. 1990. 

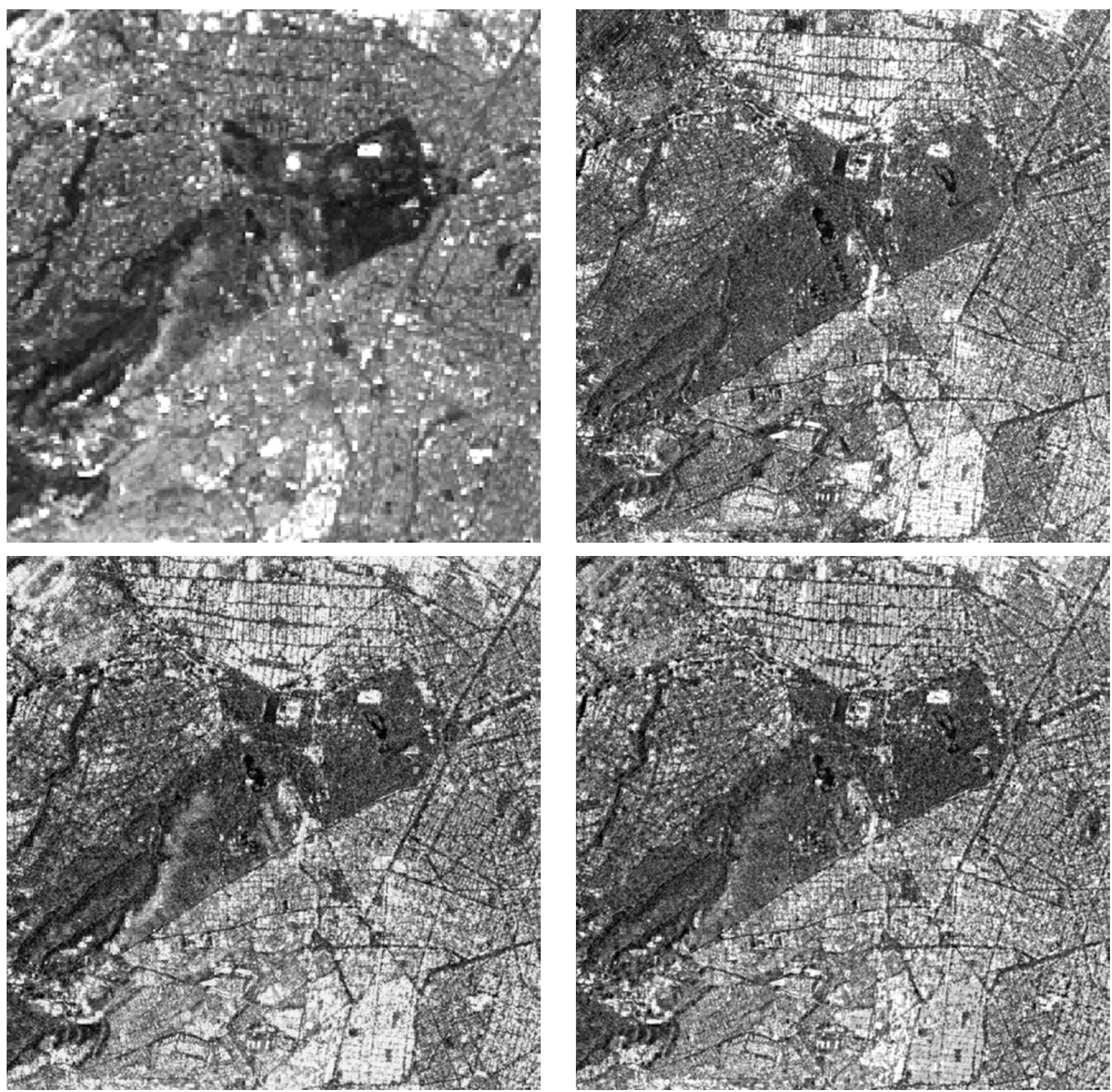

Figure 1. Upper left: LANDSAT image; upper right: RADARST image; bottom left : fused image with the Hermite transform; bottom right: fused image with the wavelet tarnsform

[6] Pohl C., van Genderen, J.L., "Multisensor image fusion in remote sensing: concepts, methods and applications", International Journal of Remote Sensing, vol 19, No, 5, pp. 823854, 1998.

[7] Porat, M. and Zeevi, "The generalized Gabor scheme of image representation in biological and machine vision," IEEE Trans. Pattern Analysis Mach, Intel., vol 10, pp. 452-467, July 1988.

[8] Watson, A. "The cortex transform: Rapid computation of simulated neural images", Comput. Vision, Graph., Image Processing, vol 39, pp. 311-327, 1987.

[9] Young, R. "Oh say, can you see?" The physiology of vision", Proc. SPIE, vol.1453, pp. 92-723, 1991.

[10] Young, R. "The Gaussian derivative theory of spatial vision: Analysis of cortical cell receptive field line-weighting profiles", General Motors Res. Labs., Rep. 4920, 1986. 